\title{
Comparison of Preemptive Effects of Celecoxib and Ibuprofen on Postoperative Pain in Addicted Patients Undergoing Lower Abdominal Surgery
}

\author{
Afshin Farhanchi ${ }^{1}$, Mahshid Nikouseresht ${ }^{2}$, Elham Khorshidi ${ }^{3}$, Pouran Hajian ${ }^{1, *}$ \\ ${ }^{I}$ Assistant Professor, Department of Anesthesiology, School of Medicine, Hamadan University of Medical Sciences, Hamadan, \\ Iran \\ ${ }^{2}$ Associate Professor, Department of Anesthesiology, School of Medicine, Hamadan University of Medical Sciences, \\ Hamadan, Iran \\ ${ }^{3}$ General Practitioner, Hamadan University of Medical Sciences, Hamadan, Iran
}

* Corresponding Author: Pouran Hajian, Department of Anesthesiology, School of Medicine, Hamadan University of Medical Sciences, Hamedan, Iran.Email: hajian@umsha.ac.ir

\begin{tabular}{|c|c|}
\hline \multirow[b]{2}{*}{$\begin{array}{l}\text { Received: } 27.04 .2017 \\
\text { Accepted: } 10.09 .2017\end{array}$} & Abstract \\
\hline & \multirow{9}{*}{$\begin{array}{l}\text { Background and Objective: Pain is a common postoperative complaint, and } \\
\text { the use of analgesics before surgical trauma can effectively prevent } \\
\text { peripheral and central sensitization. We aimed to compare the preemptive } \\
\text { effects of ibuprofen and celecoxib on post-operative pain after lower } \\
\text { abdominal surgery in addicted patients. } \\
\text { Materials and Methods: In this clinical trial, after obtaining informed } \\
\text { consent, } 114 \text { addicted patients undergoing lower abdominal surgery were } \\
\text { randomly divided into three groups of } 38 \text {. The first group was given a } 200 \\
\text { mg dose of oral celecoxib, } 400 \mathrm{mg} \text { of ibuprofen was orally administered to } \\
\text { patients in the second group, and the third group was given starch powder as } \\
\text { placebo by a nurse who prepared these drugs in the form of capsules. } \\
\text { Postoperative pain was assessed by using a } 10 \text {-cm ruler as the visual } \\
\text { analogue scale at intervals of } 1 \text { and } 6 \text { hours after surgery. Postoperative } \\
\text { opioid consumption was recorded in those periods. The obtained data were } \\
\text { analyzed using the appropriate statistical tests in SPSS software. } \\
\text { Results: Mean pain scores at } 1 \text { hour after surgery were not significantly } \\
\text { different across the three groups, whereas at } 6 \text { hours after surgery, pain } \\
\text { scores were significantly lower in the ibuprofen and celecoxib groups in } \\
\text { comparison to the placebo group (P=0.001 and P=0.005, respectively). } \\
\text { Postoperative nausea and vomiting was not significantly different among the } \\
\text { three groups. } \\
\text { Conclusion: Despite the significant difference in mean pain scores among } \\
\text { the study groups, the opioid consumption doses were not significantly } \\
\text { different among the groups. Thus, the preemptive prescription of } \\
\text { nonsteroidal anti-inflammatory drugs in addicted patient does not have any } \\
\text { noticeable effects. }\end{array}$} \\
\hline \multirow{9}{*}{$\begin{array}{l}\text { How to Cite this Article: } \\
\text { Farhanchi A, Nikouseresht M, } \\
\text { Khorshidi E, Hajian P. Com- } \\
\text { parison of Preemptive Effects of } \\
\text { Celecoxib and Ibuprofen on } \\
\text { Postoperative Pain in Addicted } \\
\text { Patients Undergoing Lower } \\
\text { abdominal Surgery. Sci J } \\
\text { Hamadan Univ Med Sci. 2017; } \\
\text { 24(3): 192-198. DOI: } 10.18869 / \\
\text { acadpub.ajcm.24.3.192. }\end{array}$} & \\
\hline & \\
\hline & \\
\hline & \\
\hline & \\
\hline & \\
\hline & \\
\hline & \\
\hline & Keywords: Abdominal Surgery; Celecoxib; Ibuprofen; Opioid Addiction; Pain \\
\hline
\end{tabular}


do): 10.18869 /acadpub.ajcm.24.3.192

مقاله يخوهشى

\title{
مقايسه اثر يِيَير انه ايبويروفن و سلكو كسيب در تسكين دردهاى بـ از عمل جراحى קايين شكم در بيماران معتاد به مواد مخدر
}

\author{
افشين فرهانجى'، مهشيد نيكوسرشت ؟، الهام خورشيدى '، يوران حاجيان ا." \\ ' ' استاديار، كروه بيهوشى، دانشكده يزشكى، دانشخاه علوم يزشكى همدان، همدان، ايران

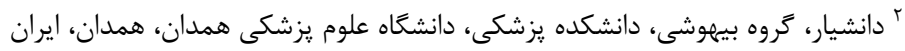

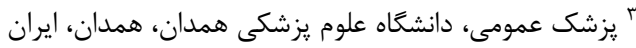

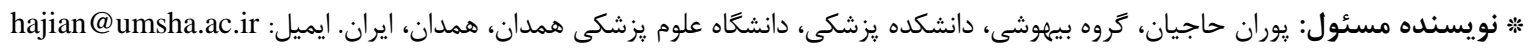

\begin{tabular}{|c|c|}
\hline 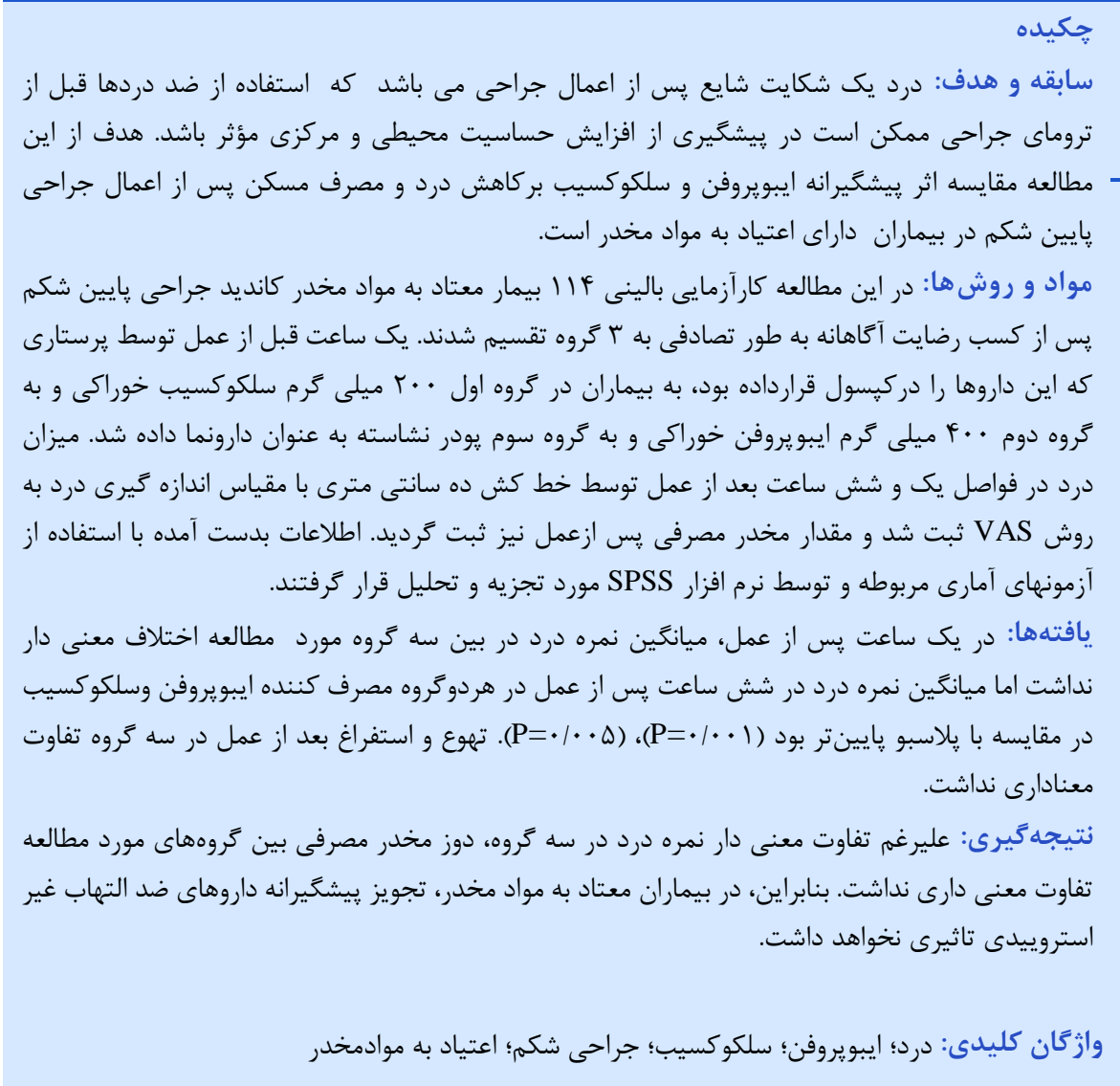 & 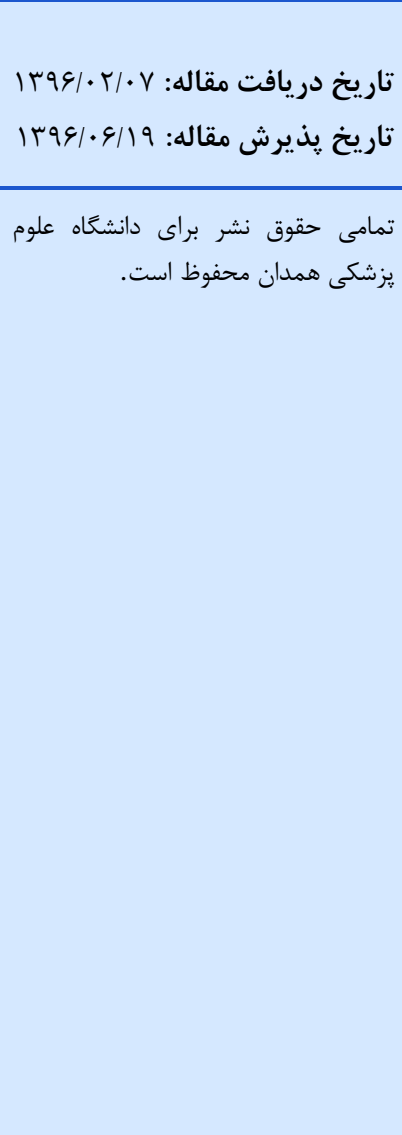 \\
\hline
\end{tabular}

مقلدمه

طريق مهار توليد Prostanoid بيش از وقوع تروماى بافتى در

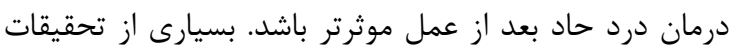

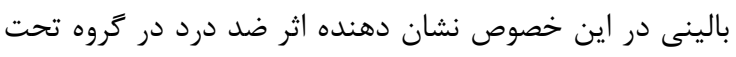

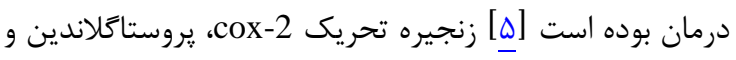
nociceptor حاد و افزايش حساسيت به درد در بافت هاى سالم محيطى نورني

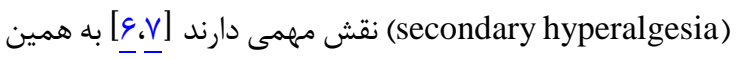

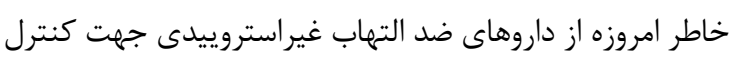
درد يس از جراحى هاى بسترى و سريايى استفاده مى شود [ــ].
درد يك شكايت شايع است كه اغلب در ارتباط با التهاب

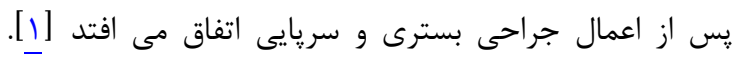

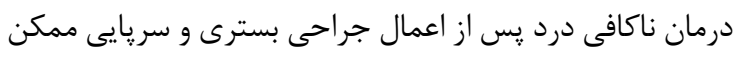

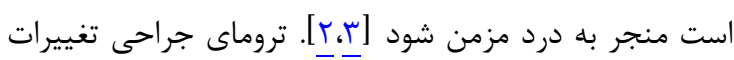

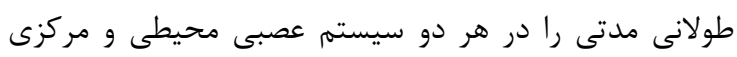

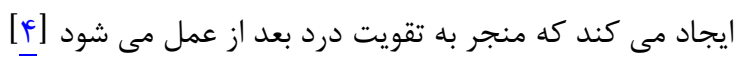

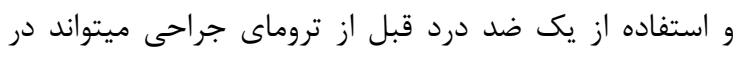

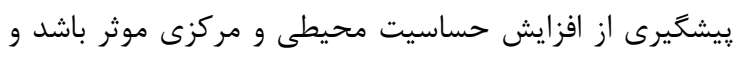

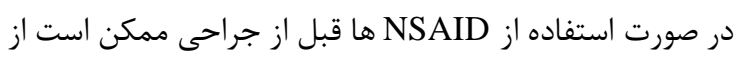


كارآزمايى بالينى به شماره رضايت آكاهانه مكتوب از بيماران مذكر معتاد به مواد مخدر

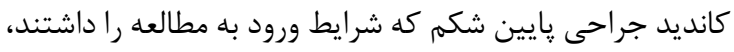

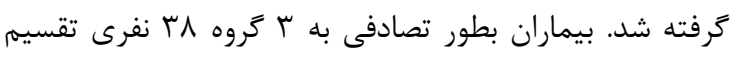

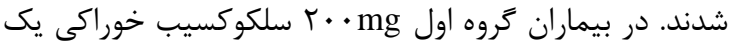

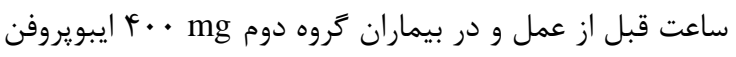

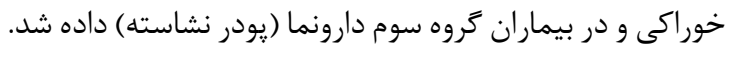

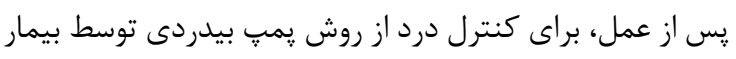

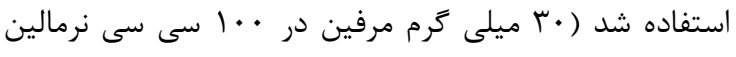

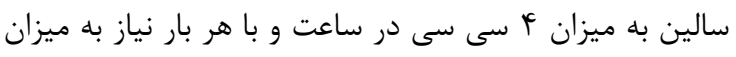

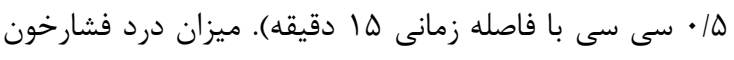

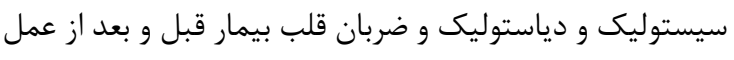

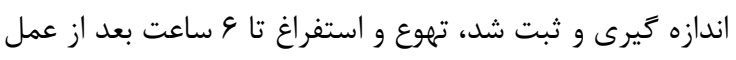

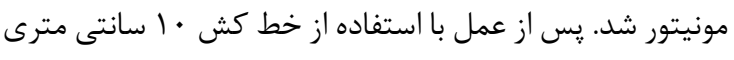

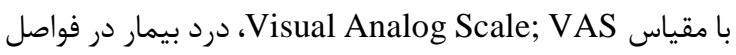
زمانى يك و شش ساعت يّ از جراحى سنجيده و ثبت شد.

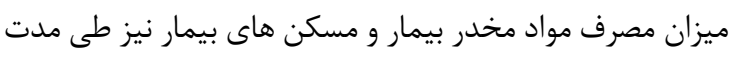
مطالعه ثبت كرديد.

براى دو سو كور كردن مطالعه، داروها طبق يك روش ريد تصادفى

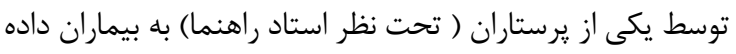

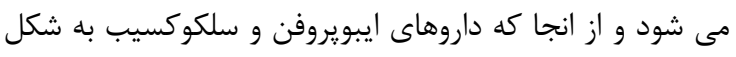
قرص و كيسول ميباشد، جهت يكسان سازى شكل دئ داروها،

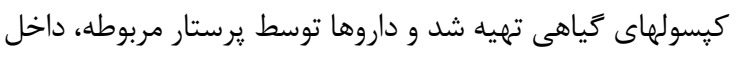

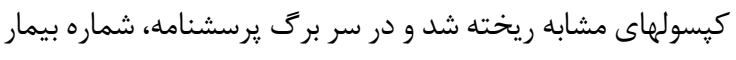

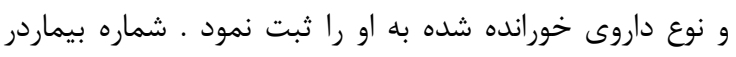

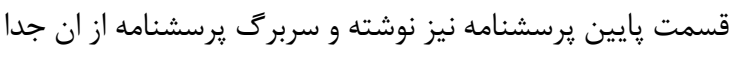

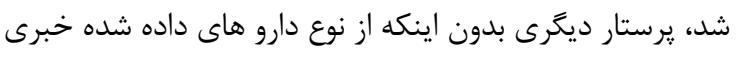

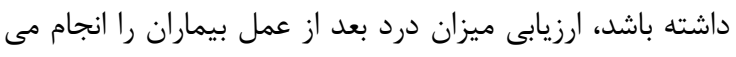

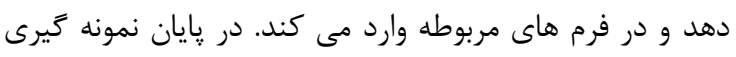

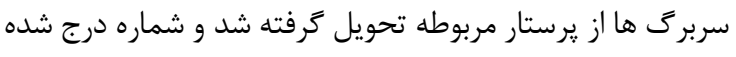

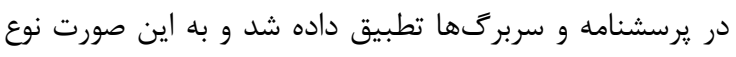

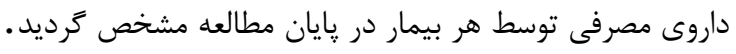

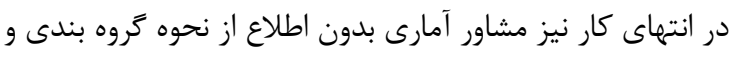

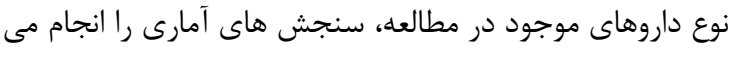

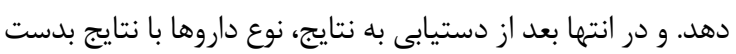

$$
\text { آمده تطبيق داده مى شود. }
$$

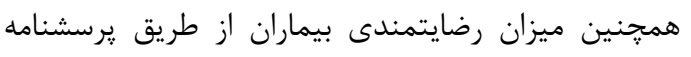

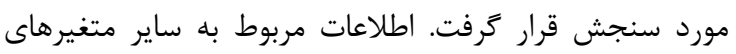

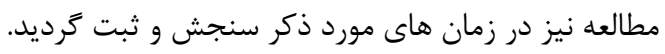

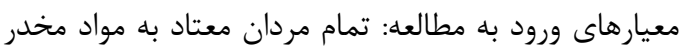

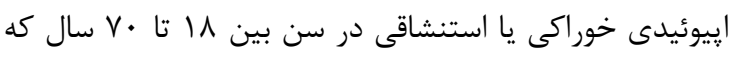
كانديد جراحى قسمت تحتانى شكمم بودند.

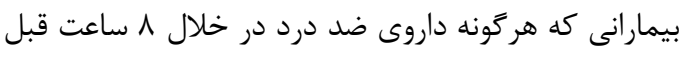

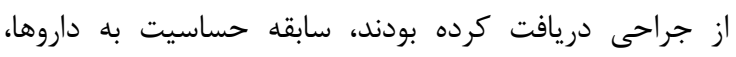

در اين بين مهار كننده هاى انتخابى cox-2 داراى مزاياى خاصى

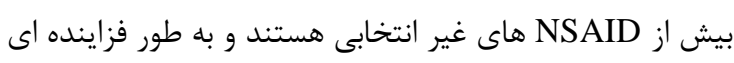

استفاده ميشوند [9].

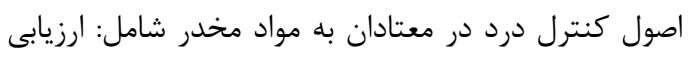

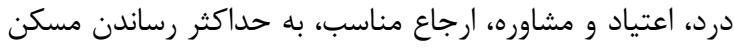
هاى غير مخدرى، درمان هاى غير دارويى و استفاده از مخدرها

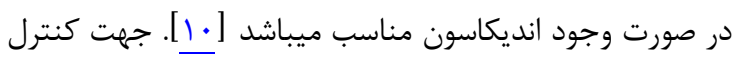

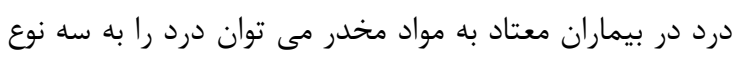

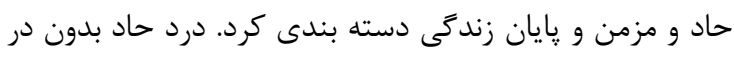

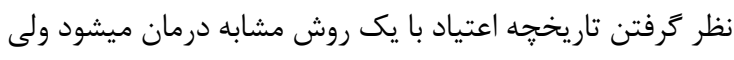

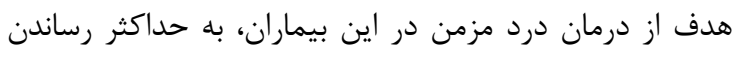
سطح عملكرد و به حداقل رساندن سو مصرف مواد مخدر است.

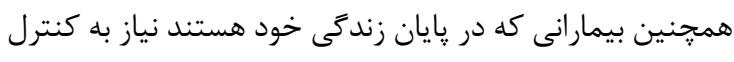

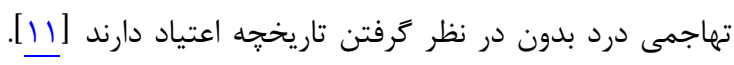

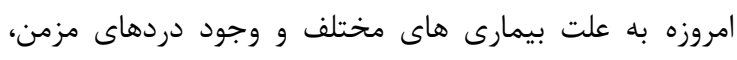

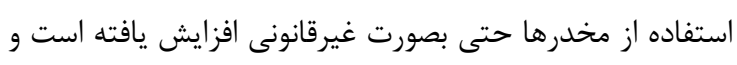

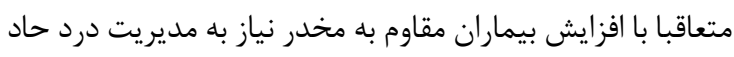

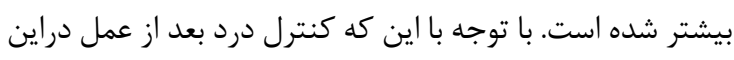

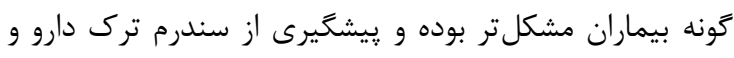
جلوكيرى از مشكلات اجتماعى و روانى و اخلاقى مربوطه نيز

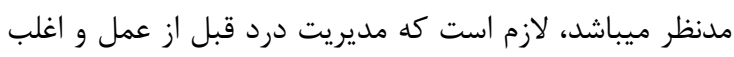

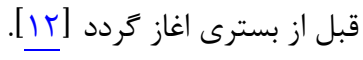

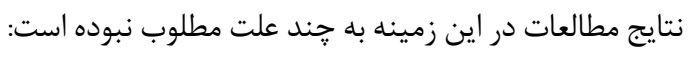
1- مصرف دوز بالاى مخدر حين عمل ميتواند مكانيسم

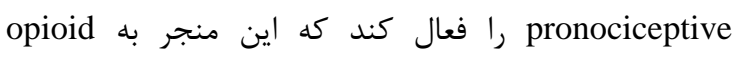
induced hyperalgesia

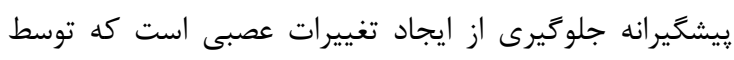

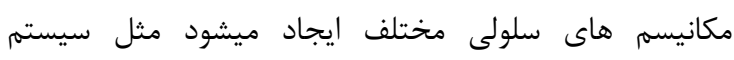
glutamatergic

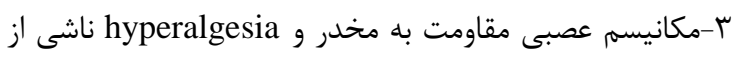

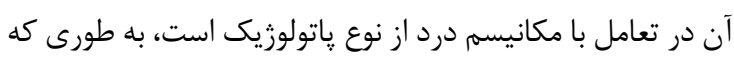

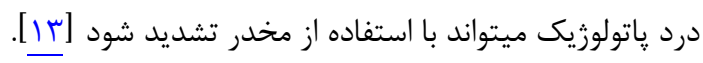

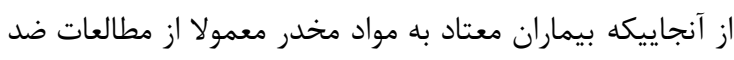

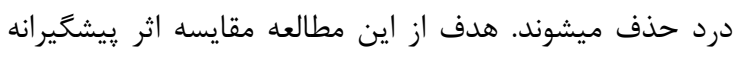

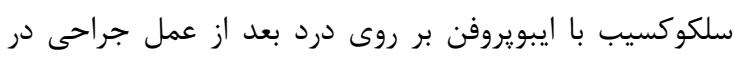
بيماران معتاد به مواد مخدر است.

\section{مواد و روشها}

اين مطالعه از نوع كارآزمايى بالينى دو سوكور تصادفى بود دود

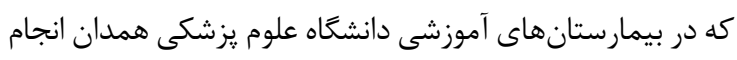

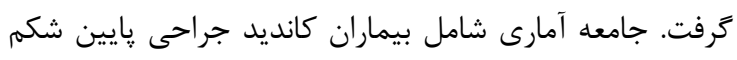
كه سابقه مصرف مواد مخدر داشتند، بود كه براساس فراس فرمول بران آمارى حجم نمونه |f || الفر محاسبه كرديد. يس از تاييد كميته اخلاق دانشخاه وثبت دمانه درديد سامانه 
آزمون آمارى نشان داد كه سه گروه مورد مطالعه از نظر

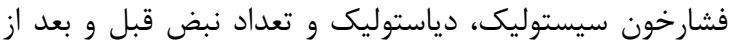
عمل از نظر آمارى اختلاف معنى دارى ندارند.

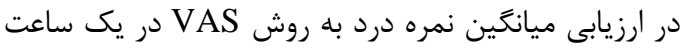

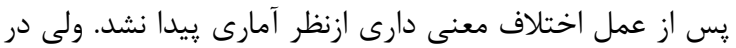

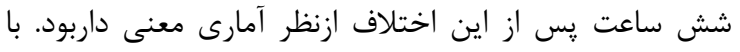

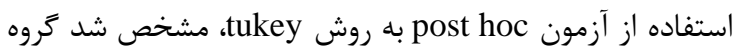
مصرف كننده ايبو بروفن و يّاسبو با هم اختلاف معنى دار دارند.

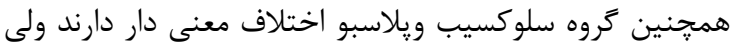

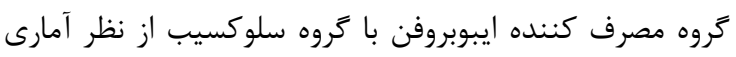
اختلاف نداشتند (جدول r).

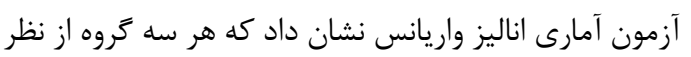
ميزان مصرف مسكن مخدرى بر حسب ميلى گرم اختلاف معنى دارى از نظر آمارى ندارند (جدول مبن).

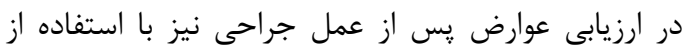

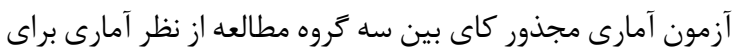

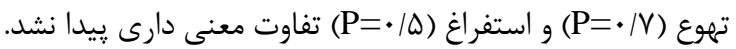

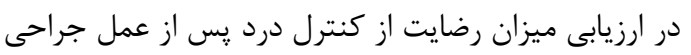

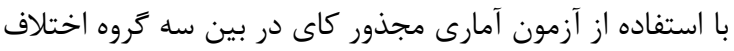

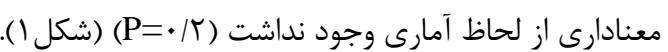

همجنين سو مصرف داروى مخدر غيرتسكينى و اعتياد به الكل،

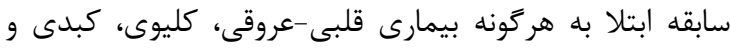

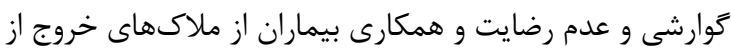
اين مطالعه بودند.

اطلاعات بدست آمده از بيماران در يرسشنامه وارد كرديد،

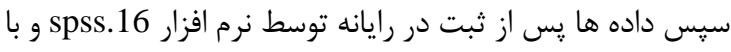

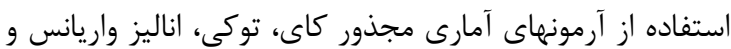

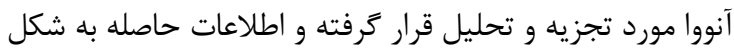

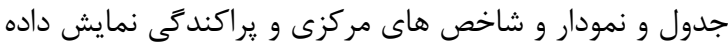

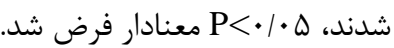
ميانگين سنى افراد در سه گروه مورد مطالعه تفاوت معنى (حسى

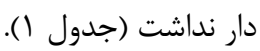
جدول ا: ميانكَين سنى افراد شركت كننده در مطالعه

\begin{tabular}{|c|c|c|c|}
\hline P ارزش P & انحراف معيار & ميانكين سن (سال) & كروه \\
\hline & $9 / 99$ & $p q / T q$ & ايبوبروفن \\
\hline$\cdot 19$ & $1 \cdot / \pi \wedge$ & $\Delta \cdot / T \wedge$ & يلاسبو \\
\hline & $q / 4 V$ & $\Delta \cdot / T \varphi$ & سلكوكسيب \\
\hline
\end{tabular}
جدول ז: ميانگين نمره درد | و و ساعت پِ از عمل در سه گروه بيماران مورد مطالعه

\begin{tabular}{|c|c|c|c|c|}
\hline PارزشP & ميانَين نمره درد & تعداد & تروه & زمان \\
\hline \multirow{3}{*}{$\cdot|V|$} & $\Delta / \cdot r$ & rı & ايبوبروفن & \multirow{3}{*}{ ا ساعت يس ازعمل } \\
\hline & $\Delta / T_{F}$ & ґ^ & يلاسبو & \\
\hline & f/qV & rᄉ & سلكو كسيب & \\
\hline \multirow[t]{2}{*}{$\cdot / \cdot \Delta$} & V/FF & rᄉ & ايبوبروفن & \multirow{3}{*}{ צ ساعت پِ ازعمل } \\
\hline & N/VG & ऍی & يلاسبو & \\
\hline$\cdot / \cdot \cdot 1$ & V/I & rᄉ & سلكو كسيب & \\
\hline
\end{tabular}

جدول ऍ: ميانكَين مصرف مخدردر 9 ساعت اول پس از عمل درسه كَروه بيماران مورد مطالعه

\begin{tabular}{|c|c|c|c|}
\hline P ارزش P & ميانگين مصرف مرفين & ت تعداد & تروه \\
\hline \multirow{3}{*}{$\cdot / \mathrm{V}$} & $N / F V$ & rی & ايبوبروفن \\
\hline & N/g & rی & يلاسبو \\
\hline & $\Lambda / 1 \Lambda$ & ґর & سلكو كسيب \\
\hline
\end{tabular}

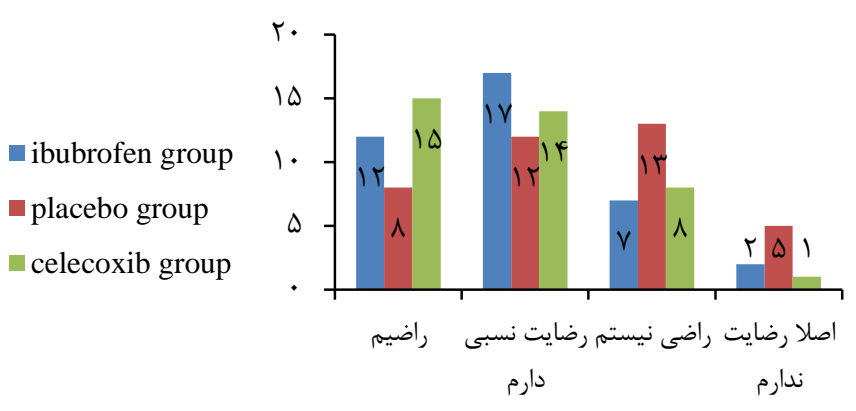

شكل ا: ميزان رضايتمندى از بيدردى پس از عمل درسه گروه بيماران مورد مطالعه 
زمان بيدردى با celecoxib در مقايسه با rofecoxib كوتاهتر

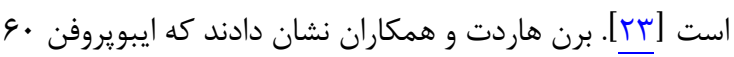

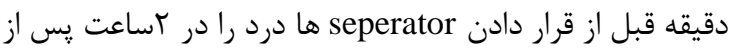

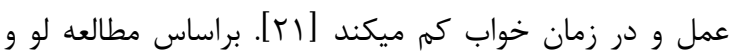

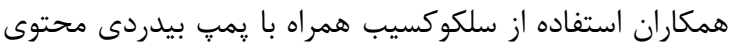

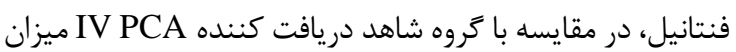

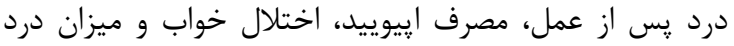

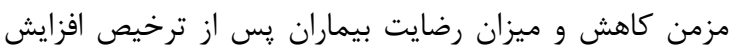

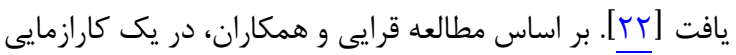

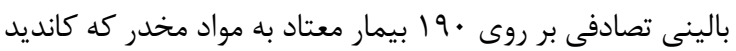

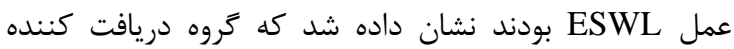

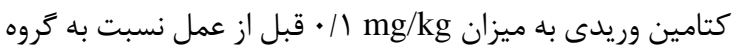

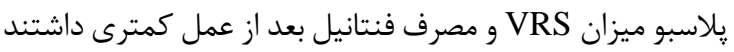

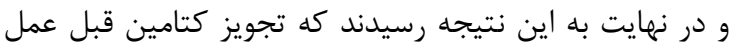

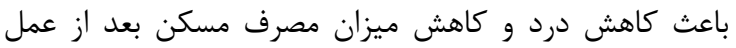

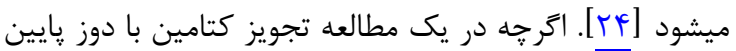

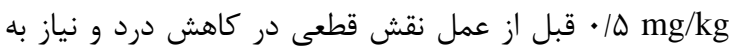
مخدر بعد از عمل در بيماران تحت كوله سيستكتومى لانى

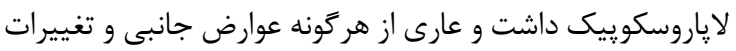

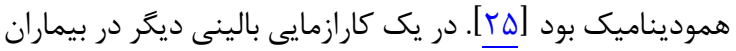
كانديد جراحى كولون نتيجه كرفتند كه تجويز دوز پايين كتامين

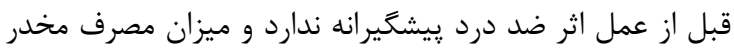

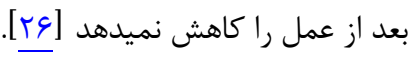

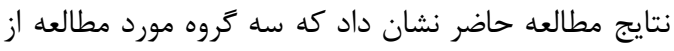
نظر فشارخون سيستوليك، دياستوليك و تعداد ضربان قلب و

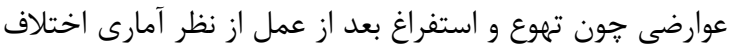

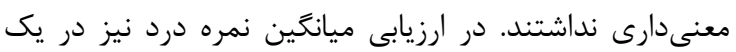

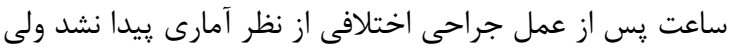

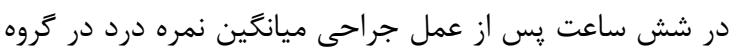
مصرفكننده ايبوبروفن و سلكوكسيب در مقايسه با يلاسبو به تهريه

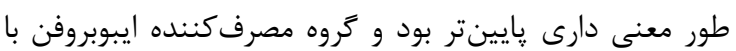

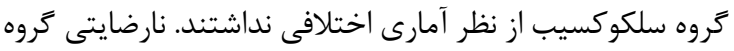

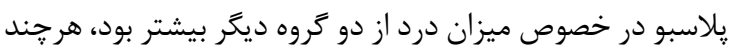

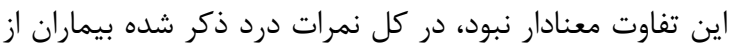

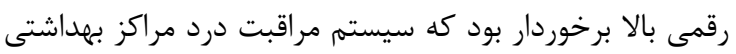

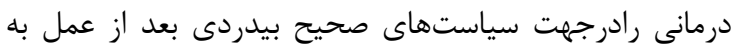
تامل وا مى دارد.

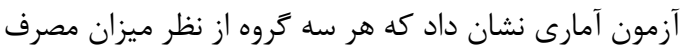

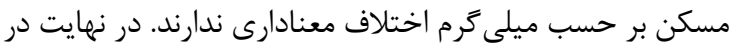

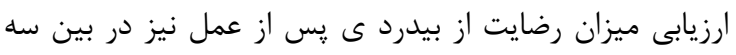

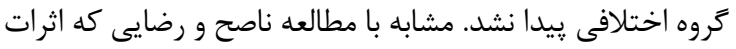

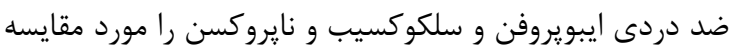

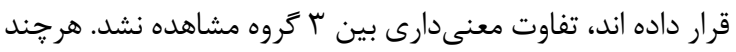

تسكين درد بعد از عمل جراحى در بيماران معتاد جالش

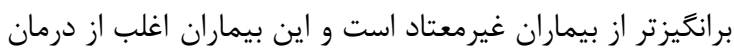

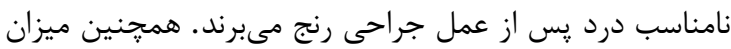

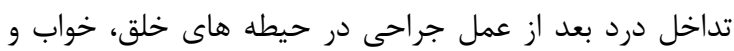

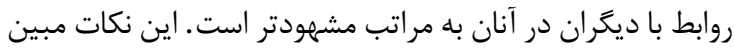

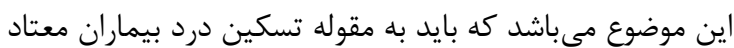

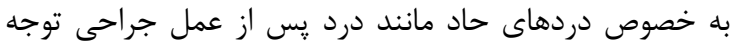

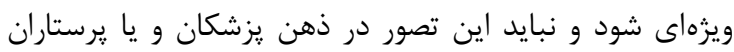

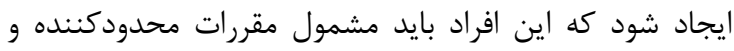

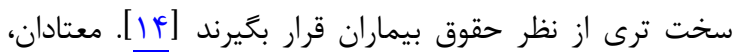
افرادى هستند كه از نظر اجتماعى رفتارشان نابهنجار جلون بلون

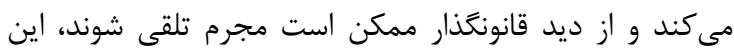

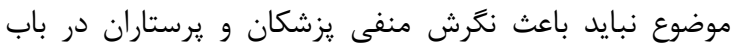

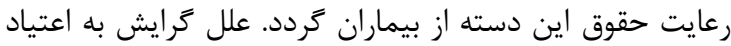
هرجه باشد وديد

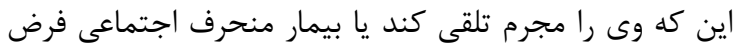

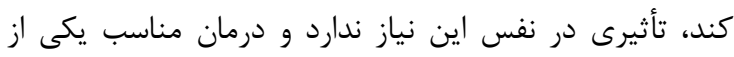

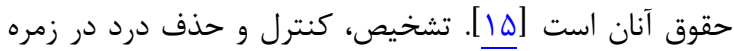

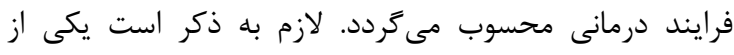
محدويتهاى يزوهش حاضر اين بود كه احتمال داشت بيماران،

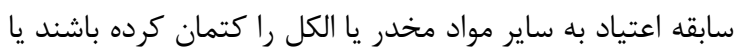

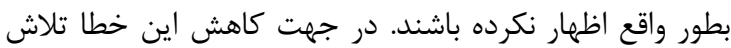

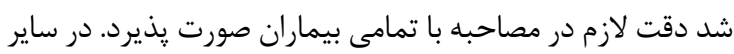
مطالعاتى هم كه بر روى بيماران معتاد انجام شده است معيار

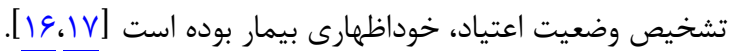

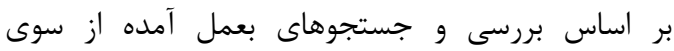
نويسند

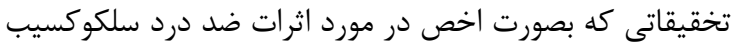

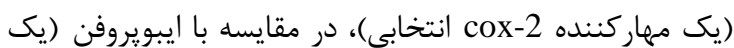

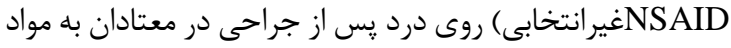

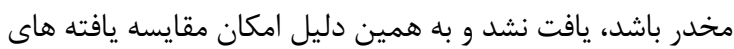

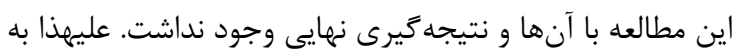

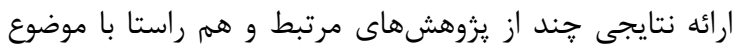
مطالعه حاضر يرداخته مىشود.

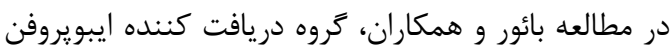

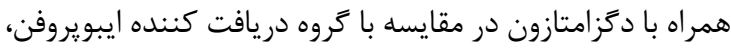

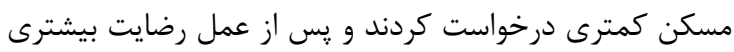

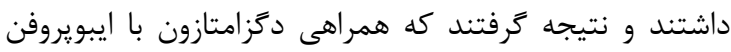

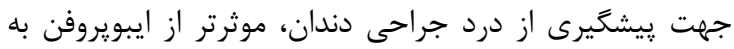

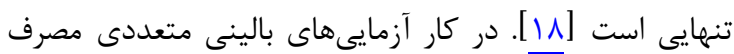

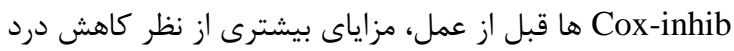

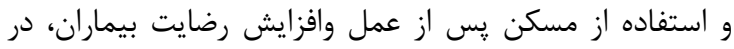

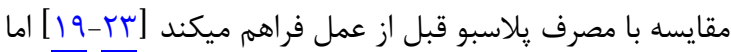


از ديخر محدوديت هاى مطالعه حاضر عدم دقت كافى

همكاران در تكميل يرسشنامه بود كه با آموزش و توجيه كامل هاصل

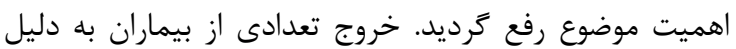
ايجاد عوارض دارو كه با جايخزين كردن نمونه حل شد و در در نهايت

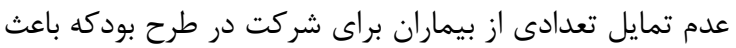
شد زمان انجام طرح نسبتا طولانى شود.

\section{نتيجه تيرى}

عليرغم تفاوت معنى دار نمره درد در سه گروه، دوز مخدر مصرفى بين گروههاى مورد مطالعه تفاوت معنى دارى نداشت.

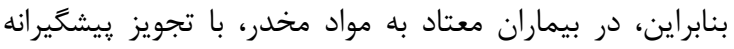

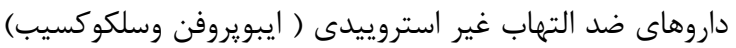
تأثير قابل توجهى رأ شاهد نخواهيم بود.

\section{تشكر و قلروانى}

اين مقاله منتج از يايان نامه دوره دكترى حرفه ایى يزشكى

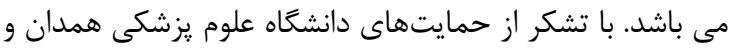

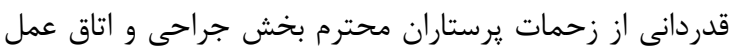

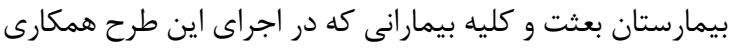

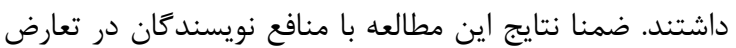
نمى باشد.

\section{REFERENCES}

1. Malmstrom K, Daniels S, Kotey P, Seidenberg BC, Desjardins PJ. Comparison of rofecoxib and celecoxib, two cyclooxygenase-2 inhibitors, in postoperative dental pain: a randomized, placebo- and active-comparator-controlled clinical trial. Clin Ther.1999;21(10):1653-63. PMID: 10566562 DOI: 10.1016/S0149-2918(99)80045-9

2. White PF, Tang J, Wender RH, Zhao M, Time M, Zaentz A, et al. The effects of oral ibuprofen and celecoxib in preventing pain, improving recovery outcomes and patient satisfaction after ambulatory surgery. Anesth Analg. 2011;112(2):323-9. PMID: 21156974 DOI: 10.1213/ANE.0b013e3182025a8a

3. Kehlet H, Jensen TS, Woolf CJ. Persistent postsurgical pain: risk factors and prevention. Lancet. 2006;367(9522):1618-25. PMID: 16698416 DOI: 10.1016/S0140-6736(06)68700-X

4. Woolf CJ, Chong MS. Preemptive analgesia: treating postoperative pain by preventing the establishment of central sensitization. Anesth Analg. 1993;77(2):362-79. PMID: $\underline{8346839}$

5. Reuben SS, Bhopatkar S, Maciolek H, Joshi W, Sklar J. The preemptive analgesic effect of rofecoxib after ambulatory arthroscopic knee surgery. Anesth Analg. 2002;94(1):55-9. PMID: 11772800

6. Fujikawa M, Ibuki T, Matsumura K, Sawa T. Inflammatory hyperalgesia: the role of the prostaglandin system in the spinal cord. Adv Neuroimmune Biol. 2012;3(2):197-207.

7. Samad TA, Moore KA, Sapirstein A. Interleukin-1betamediated induction of Cox-2 in the CNS contributes to inflammatory pain hypersensitivity. Nature. 2001; 410(6827):471-5. PMID: 11260714 DOI: $10.1038 / 35068566$

8. Ogilvie-Harris DJ, Bauer M, Corey P. Prostaglandin inhibition and the rate of recovery after arthroscopic meniscectomy: A randomised double-blind prospective study. J Bone Joint Surg Br. 1985;67(4):567-71. PMID: $\underline{3839796}$

9. Kivitz AJ, Moskowitz RW, Woods E, Hubbard RC, Verburg $\mathrm{KM}$, Lefkowith JB, et al. Comparative efficacy and safety of celecoxib and naproxen in the treatment of osteoarthritis of the hip. J Int Med Res. 2001;29(6):467-79. PMID: 11803730
اثرات ضددردى ايبويروفن و سلكو كسيب در تمامى ساعات بيشتر

از نايروكسن بودو در ساعت سوم بعد از جراحى بين گروههاى سلكو كسيب، نايروكسن و ايبويروفن+ نايروكسن تفاوت معنى دار مشاهده شد [rV][]. در مطالعه شهرتى و همكاران داروى ان استيل سيستئين با دارونما در مقايسه شد كه اختلال خواب و مشكلات تنفسى در بيماران معتاد به مواد مخدر يس از تجويز دارونما بهبود ييدا كرد ولى داروى ان استيل سيستئين در مقايسه با دارونما، سبب بهبود معنى دار كيفيت خواب و عملكرد ريوى در معتادان به مورفين در حال ترك نشد [N/[]. در مطالعه صابرى وهمكاران، مقايسه اثر ضد درد سلكو كسيب و ايبويروفن و ترامادول در كنترل درد يس از درمان ريشه دندان نشان داد كه در فواصل \&، و VT ساعت يس از درمان اختلاف آمارى معنى دارى بين گروههاى مختلف وجود نداشت [qץ]. در مطالعهاى كه ميرزايى و همكاران در آن به مقايسه قدرت تسكينبخشى دارونما و سلكوكسيب و و ودئ زلوفن با ساير مسكنهاى متداول در كنترل درد بيماران تحت درمان كانال ريشه دندان يرداختند، يافتهها نشان داد در ساعات F

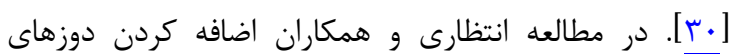
متفاوت كتامين به يمٍ بيدردى حاوى مرفين و ميدازولام در

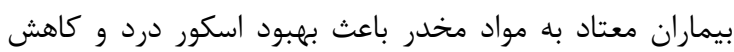
مصرف آنالززيك گرديد [1)].

\section{DOI: $10.1177 / 147323000102900602$}

10. Roberts LJ. Managing acute pain in patients with an opioid abuse or dependence disorder. Aust Prescr. 2008;31(5):1335. DOI: $10.18773 /$ austprescr.2008.075

11. Prater CD, Zylstra, RG, Miller KE. Successful pain management for the recovering addicted patient. Prim Care Companion J Clin Psychiatry. 2002;4(4):125-31. PMID: 15014719

12. Huxtable CA, Roberts LJ, Somogyi AA, MacIntyre PE. Acute pain management in opioid-tolerant patients: a growing challenge. Anaesth Intensive Care. 2011;39(5):80423. PMID: 21970125

13. Mao J. Opioid-induced hyperalgesia. $3^{\text {rd }}$ ed. New York: CRC Press; 2009. P. 174-80.

14. Koohestani H, Baghcheghi N, Rezaie K. Comparison of satisfaction with post-operative pain management and level of functional interferance in addicted and non-addicted patients. $J$ Holist Nurs Midwifery. 2014;24(3):48-55. [Persian]

15. Rahmdel M. Human rights and addicted rights. Soc Welfare Quart. 2004;4(13):13-40. [Persian]

16. American Society for Pain Management Nursing. ASPMN position: pain management in patients with addictive disease. $J$ Vasc Nurs. 2004;22(3):99-101. PMID: 15371979 DOI: 10.1016/j.jvn.2004.06.006

17. Parvizifard A, Birashk B, Atef VM, Shakeri J. Comorbidity of mood and anxiety disorders and substanceabuse among treatment-seeking addicts and normal individuals. Literary Res. 2004;2(5):45-54. [Persian]

18. Bauer HC, Duarte FL, Horliana AC, Tortamano IP, Perez FE, Simone JL, et al. Assessment of preemptive analgesia with ibuprofen coadministered or not with dexamethasone in third molar surgery: a randomized double-blind controlled clinical trial. Oral Maxillofac Surg. 2013;17(3):165-71. PMID: 22949122 DOI: $10.1007 / \mathrm{s} 10006-012-0360-7$

19. Straube S, Derry S, McQuay HJ, Moore RA. Effect of preoperative Cox-II-selective NSAIDs (coxibs) on postoperative outcomes: a systematic review of randomized studies. Acta Anesth Scand. 2005;49(5):601-13. PMID: 
15836672 DOI: 10.1111/j.1399-6576.2005.00666.x

20. Alanoglu Z, Ateş Y, Orbey BC, Türkçapar AG. Preoperative use of selective COX-II inhibitors for pain management in laparoscopic nissen fundoplication. Surg Endosc. 2005;19(9):1182-7. PMID: 16132321 DOI: 10.1007/s00464004-8254-X

21. Bernhardt MK, Southard KA, Batterson KD, Logan HL, Baker KA, Jakobsen JR. The effect of preemptive and/or postoperative ibuprofen therapy for orthodontic pain. Am $J$ Orthod Dentofacial Orthop. 2001;120(1):20-7. PMID: 11455373 DOI: $10.1067 /$ mod.2001.115616

22. $\mathrm{Lu} \mathrm{X}, \mathrm{Li} \mathrm{K}$. Multimodal effect of celecoxib on the perioperative analgesia in orthopaedic surgery. Zhong Nan Da Хие Хие Вао Yi Хие Ban.2009;34(8):815-9. PMID: 19734595

23. Reuben SS, Connelly NR. Postoperative analgesic effects of celecoxib or rofecoxib after spinal fusion surgery. Anesth Analg. 2000;91(5):1221-5. PMID: 11049912

24. Gharaei B, Jafari A, Aghamohammadi H, Kamranmanesh M, Poorzamani M, Elyassi H, et al. Opioid-sparing effect of preemptive bolus low-dose ketamine for moderate sedation in opioid abusers undergoing extracorporeal shock wave lithotripsy: a randomized clinical trial. Anesth Analg. 2013;116(1):75-80. PMID: 23223117 DOI: 10.1213/ANE.0b013e31826f0622

25. Singh H, Kundra S, Singh RM, Grewal A, Kaul TK, Sood D. Preemptive analgesia with ketamine for laparoscopic cholecystectomy. J Anaesthisol Clin Pharmacol. 2013;
29(4):478-84. PMID: 24249984 DOI: $10.4103 / 0970-$ 9185.119141

26. Nistal-Nuño B, Freire-Vila E, Castro-Seoane F, CambaRodriguez M. Preoperative low-dose ketamine has no preemptive analgesic effect in opioid-naive patients undergoing colon surgery. F1000Res. 2014;3:226. PMID: 25671084 DOI: 10.12688/f1000research.5258.1

27. Naseh M, Rezaiekalat S. Comparison of the effects of celecoxib, naproxen and ibuprofen on pain control after periodontal surgeries. J Mashhad Dent Sch. 2011;35(4):30614. [Persian]

28. Shohrati M, Shajie A, Nekuhesh L, Almasi V, Naghizadeh MM, Ghaneie M. N-acetyl cysteine effect on sleep quality and respiratory function of morphine addicts in treatment period pilot study. J Mil Med. 2010;11(4):197-201. [Persian]

29. Saberi EA, Hosseini-Goosheh M, Mirkahnooj R, Ansari H. A comparative study between the efficacy of tramadol, celecoxib and ibuprofen in pain control after root canal therapy of tooth. Zahedan J Res Med Sci. 2011;12(5):79-83. [Persian]

30. Mirzaie M, Kavosi A, Atbaie A, Moazami F, Nooribaiat S. Effect of premedication with Celecoxib and Gelofen on reduction of post-endodontic pain. J Dent Med. 2011; 24(3):172-80. [Persian]

31. Imani F, Abdollahzadeh Baghaie A. Effect of adding ketamine to the combination of morphine and midazolam in opioid tolerant patients on post-operative pain. Anesthesiol Pain. 2015;5(4):50-8. [Persian] 